\title{
Instability of strong magnetic field and neutrino magnetic dipole moment
}

\author{
Hyun Kyu Lee* \\ Department of Physics, Hanyang University University \\ Seoul, 04763, Korea \\ *E-mail: hyunkyu@hanyang.ac.kr
}

\begin{abstract}
Vacuum instability of the strong electromagnetic field has been discussed since long time ago. The instability of the strong electric field due to creation of electron pairs is one of the examples, which is known as Schwinger process. What matters are the coupling of particles to the electromagnetic field and the mass of the particle to be produced. The critical electric field for electrons in the minimal coupling is $E_{c} \sim \frac{m^{2}}{e}$. Spin $1 / 2$ neutral particles but with magnetic dipole moments can interact with the electromagnetic field through Pauli coupling. The instability of the particular vacuum under the strong magnetic field can be formulated as the emergence of imaginary parts of the effective potential. In this talk, the development of the imaginary part in the effective potential as a function of the magnetic field strength is discussed for the configurations of the uniform magnetic field and the inhomogeneous magnetic field. Neutrinos are the lightest particle(if not photon or gluon) in the "standard model", of which electromagnetic property is poorly known experimentally. Recently the observation of neutrino oscillation shows the necessity of neutrino masses. It implies that the standard model is subjected to be modified such that non-trivial electromagnetic structure of neutrino should be reconsidered although they are assigned to be neutral. And the possibility of anomalous electromagnetic form factor is an open question theoretically and experimentally. In this talk, the implication of non-vanishing magnetic dipole moment of neutrinos is also discussed: the instability of the strong magnetic field and the enhancement of neutrino production in high energy collider experiments.
\end{abstract}

Keywords: strong magnetic field, neutrino magnetic moment, Pauli coupling, instability, particle production

\section{Introduction}

There are various indications of the ultrastrong magnetic fields, $B>B_{c} \sim 10^{13} \mathrm{G}$, from astrophysical observations and terrestrial accelerator experiments and there have been continuous efforts in finding their definite evidence in Nature. Among the examples considered so far are strong magnetic fields captured on magnetar and gamma ray bursts(GRB) central engine and also created in noncentral heavy-ion collisions. Magnetars $\frac{1}{}$ are considered to be neutron stars, where strong magnetic fields of typically $10^{13} \sim 10^{15} \mathrm{G}$ are the main source of energy. Even the stronger field strength is expected inside magnetars. One of the viable models of GRB central engine is to make use the idea of tapping the rotational energy of black hole by the strong magnetic field ${ }^{2}$. It is estimated to be $\sim 10^{15} \mathrm{G}$ particularly for long and energetic bursts. In noncentral heavy-ion collisions, strong magnetic fields can also be created by the two electric currents in opposite directions generated by two colliding nuclei. it is expected that the magnetic fields in RHIC $\mathrm{Au}+\mathrm{Au}$ collisions and $\mathrm{LHC} \mathrm{Pb}+\mathrm{Pb}$ collisions can be as large as $10^{19} \mathrm{G}$ and $10^{20} \mathrm{G}$ respectively $\underline{\underline{3}}$. These field strengths are much stronger than the critical magnetic field and offer 
an interesting opportunity to study the effect of super-strong electromagnetic fields beyond the classical electrodynamics and beyond the standard model of electro-weak interaction. One of the interesting questions with given such strong electromagnetic fields is the stability of the field configuration or equivalently whether there is any instability, which leads to the particle production. Vacuum instability of the strong electromagnetic field has been discussed since long time ago. The instability of the strong electric field due to the creation of electron pairs is one of the examples, which is known as Schwinger process ${ }^{4}+10$. What matters are the coupling of particles to the electromagnetic field and the mass of the particle to be produced. The critical electric field for electron is $E_{c} \sim \frac{m^{2}}{e}$. If it is possible to imagine a charged particle lighter than the electron, the critical field can be lowered down to the field strength produced in the future high intensity laser and will be subjected to be tested in the laboratory.

The pair production of fermions in a purely magnetic field configuration is shown to be absent $\underline{\underline{5}}$. Therefore, the pair production of minimally interacting particles is considered to be a purely electric effect. However, while the minimal coupling derived by the local gauge invariance is of fundamental nature, there appear also non-minimal couplings as well in the form of the effective theory. Pauli introduced a non-minimal coupling of spin-1/2 particles with electromagnetic fields, which can be interpreted as an effective interaction of fermions with an anomalous magnetic moment $\underline{\underline{6}}$. Hence for the neutral fermions, which have no minimal coupling to electromagnetic fields, the nonvanishing magnetic moments may be the primary window through which the electromagnetic interaction of neutral fermions can be probed with the Pauli interaction.

It is known the spatial inhomogeneity of the magnetic field exerts force on the magnetic dipole moment through the Pauli interaction. It plays a similar role analogous to the electric field for the creation of charged particle pairs with the minimal coupling. The possibility of pair production of the neutral fermions in a purely magnetic field configuration with spatial inhomogeneity has been demonstrated in $2+1$ dimension 7 . The production rate in $3+1$ dimension has been calculated explicitly for the magnetic fields with a spatial inhomogeneity ${ }^{8.9}$, which can be approximated as

$$
\varpi \propto m^{4} e^{-a m^{2} /\left|\mu B^{\prime}\right|}
$$

analogues to the Schwinger process.

The instability of the particular vacuum under the strong magnetic field can be formulated as the emergence of imaginary parts of the effective potential. For uniform magnetic fields which interact with spin- $1 / 2$ fermions through the Pauli interaction ${ }^{11}$, it is found that the non-vanishing imaginary part develops for a magnetic field stronger than the critical field $B_{c}$, whose strength is the ratio of the fermion mass to its magnetic moment, $B_{c}=\frac{m}{\mu}$, 


$$
\mathcal{I}\left(V_{e f f}\right)=\frac{m^{4}}{48 \pi}\left(\frac{|\mu B|}{m}-1\right)^{3}\left(\frac{|\mu B|}{m}+3\right) .
$$

In section 2 , the calculations of the effective potential and vacuum decay rates for the neutral fermion with Pauli coupling to electromagnetic field will be reviewed.

The implication of non-vanishing magnetic dipole moment of neutrinos, the instability of the strong magnetic field and the neutrino production through the Pauli coupling in high energy collider experiment, is discussed in section 3.

\section{Imaginary part of effective potential and pair production}

The instability of the electro-weak vacuum for a strong magnetic field was discussed long time ago $\frac{15}{2}$. The one-loop effective potential considering the weak-boson $(W)$ loop is found to have imaginary part under the pure magnetic background,

$$
\mathcal{I}\left(V_{e f f}\right)=\frac{e^{2}}{8 \pi} B^{2}\left(1-\frac{m_{W}^{2}}{e B}\right) \Theta\left(B-\frac{m_{W}^{2}}{e^{2}}\right)
$$

where $m_{W}$ is the mass of $W$-boson. In the limit of $m_{W} \rightarrow 0$, eq.(3) agrees with the effective potential given by Nielson and Olesen ${ }^{16}$. It was argued that the instability could be avoided if the condensation of $W$ and $Z$ bosons $\frac{15}{}$ appears with the strong magnetic field. The basic reason for the emergence of an imaginary part for $B>\frac{m_{W}^{2}}{e^{2}}$ is that the energy eigenvalue crosses zero at $B=\frac{m_{W}^{2}}{e^{2}}$ because of the anomalous magnetic moment of $W$ boson.

One can see that there is also a level crossing of energy eigenvalue of a neutrino with Pauli interaction for a strong enough magnetic field. For a uniform magnetic field, the energy eigenvalues of the Hamiltonian are given by

$$
E= \pm \sqrt{p_{l}^{2}+\left(\sqrt{m^{2}+p_{t}^{2}} \pm \mu B\right)^{2}}
$$

where $p_{l}$ and $p_{t}$ are respectively the longitudinal and the transversal momentum to the magnetic field direction. One can see that for a magnetic field stronger than the critical field $B c=m / \mu$, the ground state with $p_{l}=p_{t}=0$ crosses the zero energy state. This indicates the possible instability of the magnetic field configuration beyond critical field strength as in electro-weak instability 18 . For a uniform magnetic field, the imaginary part of the one-loop effective action is calculated explicitly 11

$$
\mathcal{I}\left(V_{e f f}\right)=\frac{m^{4}}{48 \pi}\left(\frac{|\mu B|}{m}-1\right)^{3}\left(\frac{|\mu B|}{m}+3\right) \Theta(|\mu B|-m) .
$$

which takes the similar form as in eq. (3). It is interesting to note that the development of the imaginary part associated with a level crossing has been also demonstrated in the different $\operatorname{contexts}^{17}$. The state occupied in the negative energy sea becomes a particle state and the vacant positive energy state plunges into the negative sea to make an antiparticle state. The instability can be interpreted as 
the pair creation at the expense of the magnetic field strength. Then the particle production rate is given approximately by

$$
\varpi \sim 2 \mathcal{I}\left(V_{\text {eff }}\right)=\frac{m^{4}}{24 \pi}\left(\frac{|\mu B|}{m}-1\right)^{3}\left(\frac{|\mu B|}{m}+3\right) \Theta(|\mu B|-m) .
$$

For a nonuniform magnetic field, the inhomogeneity of the magnetic field coupled directly to the magnetic dipole moment plays an interesting role analogous to the electric field for a charged particle: The non-zero gradient of the magnetic field can exert a force on a magnetic dipole moment. Then the vacuum production of neutral fermions with a non-zero magnetic moment in an inhomogeneous magnetic field is possible. As a simple example, a static magnetic field configuration with a constant gradient, $B^{\prime}=d B_{z} / d x$, along x-direction,

$$
B_{z}(x)=B_{0}+B^{\prime} x .
$$

has been considered. It is not necessary to consider an infinitely extended everincreasing magnetic field to meet the linear magnetic field configuration. Because the particle production rate density is a local quantity, it is sufficient to have a uniform gradient magnetic field in the Compton wavelength scale of the particle. The imaginary part of the one-loop effective potential is calculated in the integral form 11 ,

$$
\begin{aligned}
\mathcal{I}\left(V_{e f f}\right)= & -\frac{m^{4}}{4 \pi^{2} \lambda \kappa} \int_{0}^{\infty} \frac{d v}{v^{2}}\left[\sqrt{v \operatorname{coth} v} F\left(\frac{\lambda}{\kappa} \tanh v, \lambda v\right)-\frac{1}{2} \cos \lambda v-\frac{\lambda v}{12 \kappa} \sin \lambda v\right] \\
& -\frac{m^{4}}{4 \pi^{2} \lambda^{2}} \int_{0}^{\infty} \frac{d v}{v^{3}}\left[(v \operatorname{coth} v)^{3 / 2}-1-\frac{v^{2}}{2}\right] \sin \lambda v
\end{aligned}
$$

where

$$
\begin{aligned}
v & =s \mu B^{\prime}, \quad \lambda=\frac{m^{2}}{\left|\mu B^{\prime}\right|}, \quad \kappa=\frac{m^{2}}{|\mu B|^{2}}, \\
F(a, b) & =\int_{0}^{1} d \xi(1-\xi) \cos \left(a \xi^{2}-b\right)
\end{aligned}
$$

Numerically it is found that it can be fitted to an analytic expression 11 ,

$$
\mathcal{I}\left(V_{e f f}\right) \propto m^{4} e^{-b \frac{m^{2}}{\left|\mu B^{\prime}\right|}},
$$

which is decreasing exponentially with respect to the inverse of the field gradient. The qualitative feature of this result can be compared with the expression obtained recently by Gavrilov and Gitman $\stackrel{9}{ }$.

\section{Neutrino magnetic dipole moment and its implications}

Neutrinos are the lightest particles(if not photon or gluon) in the "standard model", of which electromagnetic property is poorly known experimentally. Moreover the observation of neutrino oscillation shows the necessity of neutrino masses. It implies 
that the standard model is subjected to be modified such that non-trivial electromagnetic structure of neutrino should be reconsidered although they are assigned to be neutral. While there is no observation which is not consistent with the electriccharge neutrality of neutrinos, the possibility of anomalous electromagnetic form factor is an open question theoretically and experimentally 13.

So far there is no experimental evidence of the magnetic dipole moment of the neutrinos but one can not simply rule out the magnetic moment just because of the electrical neutrality. For example, in a minimal extension of the standard model to incorporate the neutrino mass the anomalous magnetic moment of a neutrino is known to be developed in one loop calculation $\frac{19}{}, \mu_{\nu}=\frac{3 e G_{F}}{8 \sqrt{2} \pi^{2}} m_{\nu}$. Here one can notice that the non-zero mass is essential to get a non-vanishing magnetic moment. There are strong evidences from the neutrino oscillation observations that neutrinos have nonzero-masses 20 . However, the simple extensions of the standard model can give only much smaller magnetic moment, $\sim 10^{-20} \mu_{B}$ if we take the neutrino mass as $1 \mathrm{eV}$, than the experimental bound. GEMMA collaboration 21 observed that the neutrino magnetic moment is bounded from above by the value $\mu_{\nu}<2.9 \times 10^{-11} \mu_{B}$ and the bound obtained from Borexino 22 data gave $\mu_{\nu} \leq$ $3.1 \times 10^{-11} \mu_{B}$. Since a wide range of neutrino magnetic moments is possible up to the current laboratory upper limit beyond standard model, it is interesting to investigate the phenomenological consequences of the nonvanishing magnetic moment up to the current bounds, which may constrain the bound tighter than the direct measurements of the neutrino magnetic dipole moment.

For the vacuum instability, the typical field strength(or critical field strength) is given by $B_{c}=m_{\nu} / \mu_{\nu}$. Taking the possible magnetic moment to be as large as the experimental upper bound $\mu_{\nu}=10^{-11} \mu_{B}$ and the mass of the neutrino $m_{\nu} \sim 10^{-2} \mathrm{eV}$ constrained by the neutrino oscillations, the critical field strength is estimated to be $B_{c} \sim 10^{17} \mathrm{G}$, which is not far from the field strength inferred on the magnetar surface and might be possible inside the magnetar. With vacuum instability, the particle production rate per unit time per unit volume is typically of order $m_{\nu}^{4}$ for the critical field strength. As a possible environment, let us consider the pair creation of neutrinos with non-zero magnetic dipole moments from the very strongly magnetized compact objects with $B=B_{c}$ and radius $R_{B}$. With $R_{B}^{3}$ as an effective volume of the magnetosphere, the production rate can be estimated ${ }^{23}$ as

$$
\varpi_{\nu} \sim 10^{39}\left(\frac{m_{\nu}}{10^{-2} e V}\right)^{4}\left(\frac{B}{B_{c}}\right)^{4}\left(\frac{R_{B}}{10 k m}\right)^{3} / s .
$$

Using the currently estimated masses of neutrinos the corresponding luminosity is estimated to be $L \sim 10^{25} \mathrm{erg} / \mathrm{s}$ assuming $E_{\nu} \sim m_{\nu}$, which is much weaker than the X-ray luminosity of magnetars, $L_{X} \sim 10^{35} \mathrm{erg} / \mathrm{s}$. However, since the time scale is much longer than the Schwinger process by a factor of $\left(m_{e} / m_{\nu}\right)^{4}$, it can be a continuous source of neutrinos. A larger luminosity for observation can be easily obtained with a massive particle but the magnetic moment should be increasing to keep the critical field strength, which significantly constrains models for particles 
involved. For the vacuum instability beyond critical field strength in case of the uniform magnetic field, the interpretation of vacuum instability as the neutrino pair production may not be the whole story. It is because there may be a possibility that the instability could be avoided if a nontrivial condensation $\underline{\underline{15}}$ develops along with the critical magnetic field, which is subjected to further discussion.

The pair production of neutrinos in collider experiments can be enhanced if endowed with the nonvanishing magnetic dipole moment. The pair production of neutrinos are expected from the annihilation of charged particles in colliding experiments through photon channel ${ }^{12}$ in addition to the weak boson channel in the standard model. One of the interesting features of Pauli coupling is that the angular distribution of pair creation peaks at $\theta=\pi / 2$. It is compared to the angular distribution of the standard model process, minimum at $\theta=\pi / 2$ but maximum for $\theta=0$ and $\pi$. For the Majorana neutrinos, the pairs with different flavors can be produced due to the transit magnetic moment couplings. The the angular distribution peaks at $\theta=\pi / 2$ with respect to the beam direction, which is the enhancement of the neutrino production at right angle in contrast to weak Z-channel. It is interesting to note that the flavor mixing angle can be inferred if the flavor correlation in pairs can be measured in the future experiments $\frac{14}{}$. The detectors located around the right angle to the beam direction can measure the back-to-back correlations in the pair production, where the production rate is supposed to be maximum. Most of the detectors are using electromagnetic triggers at the end stations, which implies corresponding charged leptons $\left(l_{\alpha}\right.$ and $\left.l_{\beta}\right)$, for example electrons or muons, are those to be detected finally. The Majorana pairs in mass eigenstates, $\nu_{1}$ and $\nu_{2}$, are produced through the Pauli coupling and interact weakly with other particles to produce charged leptons to be detected. To simplify the situation let us consider only two mass eigenstates which are mixed states of weak eigenstates, $\nu_{\alpha} \nu_{\beta}$,

$$
\nu_{1}=\cos \delta \nu_{\alpha}+\sin \delta \nu_{\beta}, \quad \nu_{2}=-\sin \delta \nu_{\alpha}+\cos \delta \nu_{\beta}
$$

where $\delta$ is the mixing angle. Now consider two targets A and B placed at the opposite side of the center of mass at the right angle to the beam direction. At the targets the neutrinos can produce the corresponding charged leptons via the charged current weak interaction(W-boson). The back-to-back correlation, $\mathrm{R}$, defined by the product of the detection rates of $\alpha$ type leptons at the target $\mathrm{A}$ and $\beta$ type leptons at the target $\mathrm{B}$, can be given by

$$
R=\frac{1}{2}\left(1+\cos ^{2} 2 \delta\right) .
$$

Since the correlation $R$ turns out to be dependent only on the mixing angle, $\delta$, it may serve as a clean measurement of mixing angles in the collider experiments. For example the maximum value, $R=1$, is obtained for the cases of no-mixing $(\delta=0)$, $R=1 / 2$ for the maximal mixing $(\delta=\pi / 4)$.

These features are quite different aspect from standard model, which can be clearly distinguished in the high energy collider experiment $\underline{14}$. Sine the strength of 
Pauli coupling to the photon is determined by the neutrino magnetic dipole moment, which is very small, the pair creation cross section through photon channel by the Pauli coupling is smaller that that of weak boson $(Z)$ channel until the center of mass energy of the colliding particle is sufficiently high. To get some idea of the energy scale for which the Pauli coupling becomes dominant, we can define the energy scale, $E_{0.1}$, for which the total cross section becomes $10 \%$ of the standard model,

$$
E_{0.1}=10^{3}\left(\frac{10^{-11} \mu_{B}}{\mu_{\nu}}\right) T e V .
$$

It is a bit higher than LHC energy, $E_{C M} \sim 10 \mathrm{TeV}$ and also higher than the GZK cut-off energy of ultra high energy cosmic rays, $E_{C M} \sim 100 \mathrm{TeV}$. So to see the distinguished feature from standard model, the higher energy collider experiments than present LHC seem to be needed.

The magnetic field is expected be created by the two electric currents in opposite directions generated by two colliding nuclei. It turns out the field strength may be very large as $10^{19}-10^{20} \mathrm{G}$ at RHIC and LHC. It gives $10^{4}$ times stronger Pauli coupling than expected at the surface of the magnetars. The magnetic vacuum instability due to magnetic dipole moment of neutrino can be much more enhanced. How to identify experimental observables corresponding to the vacuum instability and particle productions that are sensitive to the neutrino magnetic dipole moment may be an interesting and challenging subject to be discussed.

\section{Acknowledgments}

The author would like to thank Dmitry Gitman, Sang Pyo Kim and Yongsung Yoon for useful comments and discussions.

\section{References}

1. S.Mereghetti, J.Pons, A. Melaos, Space Science Review, 191, 1315(2015); McGill Catalogue,http://www.physics.mcgill.ca/ pulsar/magnetar/main.html

2. H.K. Lee, R. Wijers and G.E. Brown, Phys. Rep. 325, 83 (1999).

3. X-G. Huang, Rep. Prog. Phys. 79, 076302 (2016).

4. J. Schwinger, Phys. Rev. 82, 664(1951).

5. See for example, G. Dunne and T.M. Hall, Phys. Lett. B419, 322(1998); Phys. Rev. D60, 065002(1999).

6. W. Pauli, Rev. Mod. Phys. 13, 203(1941).

7. Q.-G. Lin, J. Phys. G. 25, 1793(1999).

8. H.K. Lee and Y.S. Yoon, JHEP 0603, 078(2006).

9. S.P. Gavrilov and D.M. Gitman Phys. Rev. D 87, 125025 (2013).

10. S.P. Kim, H.K. Lee and Y.S. Yoon, Phys.Rev.D82,025015(2010) and references therein.

11. H.K. Lee and Y.S. Yoon, JHEP 0703,086 (2007). 
12. Y.M. Koh, H.K. Lee, W-G. Paeng and Y. Yoon, J. Korean Phys. Soc. 56, 1884 (2010).

13. C. Giunti and A. Studenkin, Rev. Mod. Phys. 87, 531(2015).

14. H.K.Lee, Phys. Rev. D84, 077302 (2011).

15. J. Ambjorn, R.J. Hughes and N.K. Nielson, Ann. Phys.(NY), 150, 92(1983):J. Ambjorn and P. Olesen, Nucl.Phys.B 330, 193(1990) and references therein.

16. N.K. Nielson and P. Olesen, Nucl. Phys. B 144, 376(1978).

17. N. Graham and R.L. Jaffe, Phys.Lett. B435, 145 (1998).

18. H.K. Lee and Y. Yoon, Mod. Phys. Lett. A 22, 2081(2007).

19. K. Fujikawa and R. Shrock, Phys. Rev. Lett. 45, 963 (1980).

20. See for example, D. Forero, M. Tortola, J. Valle, Phys. Rev. D 90, 093006(2014).

21. A.G. Beda, V.B. Brudanin, V.G.Egorov, D.V. Medvedev et al., Adv. High Energy Phys. 2012, 350150(2012).

22. B.C. Canas, O.G. Miranda,A. Parada, M. Tortola and J.W.F. Valle, Physics Letters B 753, 191(2016)

23. H.K. Lee and Y. Yoon, Mod. Phys. Lett. A 23, 17(2008). 